Title:

How specific is the MRI appearance of supratentorial atypical teratoid rhabdoid tumors?

Authors:

Au Yong, Kong Jung, MB CHB, MRCS, FRCR, MBA (1)

Jaremko, Jacob L, MD, PhD, FRCPC (1)

Jans, Lennart, B. O, MD, PhD (2)

Bhargava, Ravi, MD, FRCPC, FRCPC(1)

Coleman, Lee T, MB CHB, FRANZCR (3)

Mehta Vivek, BA, MD, MSc, FRCSC, FACS (4)

Ditchfield, Michael Ronald, MBBS, MD, FRANZCR (5)

(1) Department of Radiology and Diagnostic Imaging, University of Alberta Hospital, 8440-112 Street NW, Edmonton, AB, Canada T6G 2B7, Tel +1.780.407.6907, fax +1.780.407.3853

Email:kong.auyong@gmail.com,jjaremko@ualberta.ca,Ravi.Bhargava@albertahealthservices.ca

(2) Department of Radiology and Medical Imaging, Ghent University Hospital, De Pintelaan 185, 9000 Gent, Belgium, Tel 0032 485.33.06.33 ("Gent" is NOT a typographical error in the address)

Email: lennartjans@hotmail.com

(3) Department of Radiology and Pediatrics, University of Melbourne and Murdoch Children's Research Institute, Melbourne. Medical Imaging, Royal Children's Hospital, 2nd floor, Main Block Flemington Rd., Parkville VIC 3052, Tel +03 9345 5255, fax +03 93456694

Email: Lee.Coleman@rch.org.au

(4) Department of Neurosurgery, University of Alberta Hospital, 8440-112 Street NW, Edmonton, AB, Canada T6G 2B7, Tel +1.780.407.8346, fax +1.780.407.7293

Email: Vivek.Mehta@albertahealthservices.ca

(5) Diagnostic Imaging, Monash Children's and Monash University, Monash Medical Centre, Level 2 (Ground floor), Monash Medical Centre, 246 Clayton Road, Clayton, 3168, Victoria. Tel +61 395942010

Email: michael.ditchfield@southernhealth.org.au 
Original article

\section{How specific is the MRI appearance of supratentorial atypical teratoid} rhabdoid tumors?

\section{Kong Jung Au Yong}

Jacob L. Jaremko

Lennart Jans

Ravi Bhargava

Lee T. Coleman

Vivek Mehta

Michael R. Ditchfield

K. J. Au Yong ( $\bowtie)$, J. L. Jaremko, R. Bhargava

Department of Radiology and Diagnostic Imaging

University of Alberta Hospital

8440-112 Street NW

Edmonton, Canada T6G 2B7

e-mail:kong.auyong@gmail.com

L. Jans

Department of Radiology and Medical Imaging

Ghent University Hospital

Gent, Belgium

L. T. Coleman

Department of Radiology and Pediatrics

University of Melbourne and Murdoch Children's Research Institute

Melbourne, Australia

Medical Imaging, Royal Children's Hospital

Parkville, Australia

V. Mehta

Department of Neurosurgery

University of Alberta Hospital

Edmonton, Canada 
M. R. Ditchfield

Diagnostic Imaging

Monash Children's and Monash University

Monash Medical Centre

Clayton, Australia 


\begin{abstract}
Background Supratentorial atypical teratoid rhabdoid tumor (ATRT) in many cases has a distinctive appearance on post-gadolinium MRI.

Objective We sought to determine whether this is a unique appearance allowing ATRT to be accurately distinguished from other types of pediatric supratentorial tumors.

Materials and methods Retrospective review of all available preoperative MRI of pediatric supratentorial tumors at two tertiary children's hospitals, and systematic literature review of case series and reports describing the MRI imaging appearances of supratentorial ATRT.

Results We had 61 supratentorial tumors, including 32 gliomas, 6 ATRT, 8 ependymomae, 6 gangliogliomae, 2 pilomyxoid astrocytomas, 3 primitive neuro-ectodermal tumors, 2 choroid plexus papillomae, and 2 meningiomae. ATRT presented in significantly younger patients than astrocytomas (mean age 2.6 years vs. 9.9 years, $P<0.05$ ). The visual pattern of a thick, wavy (irregular) heterogeneously enhancing wall around a cystic centre was seen in 5/6 (83\%) ATRT and only $3 / 55(5.4 \%)$ other tumors $(P<0.0001)$, for specificity $\mathrm{SP}=0.95$, sensitivity $\mathrm{SN}=0.83$, positive predictive value $=0.63$, negative predictive value $=0.95$.

Conclusion A supratentorial tumor with a thick, wavy (irregular) heterogeneously enhancing wall surrounding a central cystic region is suggestive of ATRT in the appropriate clinical setting, especially in a child of preschool age.
\end{abstract}

Keywords Supratentorial tumor, Atypical teratoid rhabdoid tumor, MRI, Brain tumor 


\section{Introduction}

Atypical teratoid rhabdoid tumor (ATRT) is a highly aggressive tumor that is also a relatively new pathological diagnosis, first identified in 1987 [1, 2], pathologically resembling malignant rhabdoid tumors of the kidneys [3]. Previously, tumors of this type were often grouped with primitive neuroectodermal tumors (PNET) [4]. Because the immunohistochemical phenotype of ATRT varies widely [2, 5-7], the diagnosis is based on absence or inactivating mutation of a tumor-suppressor gene on chromosome 22 (hSNF5/INI1), or absence of the gene products [8]. Location of ATRT within the brain varies and affects prognosis. Infratentorial ATRT is particularly aggressive: In one series, patients with infratentorial ATRTs were significantly younger at diagnosis (median age 2.4 years supratentorial vs. 0.9 year infratentorial), had more metastases (supratentorial 3/26, infratentorial 9/27) and shorter survival (3-year overall survival $38 \%$ supratentorial vs. 5\% infratentorial) [9]. Relative incidence is unclear: in case series, infratentorial ATRT has been reported to be more common than supratentorial ATRT [10], equal in incidence $[9,11]$, or less common $[12,13]$.

The radiology literature on ATRT consists of isolated case reports and small case series. Most authors report nonspecific imaging features at computed tomography (CT) and magnetic resonance imaging (MRI), such as enhancement and heterogeneous T2 signal [2, 13-19]. Two studies noted a typical appearance of supratentorial ATRT, involving a thick, wavy (irregular) heterogeneously enhancing wall around internal cystic areas [19-20]. We could find no study dedicated to the imaging characteristics of supratentorial ATRT, and none testing whether this appearance is, in fact, specific for this tumor type. Accordingly, we searched the English literature for available cases of supratentorial ATRTs with imaging findings, and reviewed our own series of supratentorial tumors at two large pediatric hospitals. 


\section{Materials and methods}

This is a multicenter study involving two tertiary referral pediatric hospitals, the Royal Children's Hospital (Melbourne, Australia) and Stollery Children's Hospital (Edmonton,

Canada). The study was approved by each hospital's Human Research Ethics Committee. Patients' consent was not required for this retrospective study with unidentified subjects. We retrospectively reviewed imaging and pathology data of children with supratentorial tumors at these sites. We searched each hospital neurosurgical database for all cases of ATRT between 2000 and 2010. As none was found prior to 2006, we then examined initial pre-resection imaging, pathology reports and demographic data for all children who had surgical biopsy or resection of a tumor located in the cerebral hemispheres, lateral or third ventricle, except the pineal area, from 2006 to 2010 .

\section{Literature review}

We searched PUBMED/MEDLINE (1948 to November 2011) for keywords including "ATRT," "malignant rhabdoid," "teratoid," and then the MeSH terms originally used to index the papers retrieved by those searches. Further studies were located through manual search of citations in the retrieved papers. Studies reporting one or more cases of pediatric supratentorial ATRTs with description of imaging findings were included. Potentially or definitely relevant studies were assessed in consensus by two pediatric radiologists, who extracted data including demographics, tumor location, preoperative imaging features and presence of metastasis.

MRI protocol 
All MRI was performed in routine clinical care using 1.5-T or 3-T scanners at each site. Standard spin-echo T1- and T2-weighted, and post-gadolinium T1-weighted images were available on all patients. Diffusion-weighted imaging, when performed, used echo-planar base sequence with gradient fields of $b=0,500$ and $1,000 \mathrm{~s} / \mathrm{mm} 2$. Proton spectroscopy was infrequently available in this data.

Anatomical imaging analysis

Images were assessed by two pediatric radiologists blinded to pathological diagnosis, after planning with a senior pediatric radiologist. Discordances between the two were resolved by consensus.

Pathological examination

Examination, diagnosis and histological grading of specimens were performed at each hospital by a neuropathologist. The reports produced in routine clinical care were reviewed. For each ATRT, the presence of rhabdoid cells, necrosis and hemorrhage, as well as immunohistochemical staining for BAF47 (INI-1), was documented. INI-1 antibody staining correlates with molecular findings in ATRT and can confirm the histological diagnosis.

\section{Statistical analysis}

Basic descriptive statistics were computed in Microsoft Excel (Redmond, WA, USA, v. 2007), with results reported as mean $+/$ - standard deviation (SD). Using level of significance $P=0.05$, we tested differences between means of continuous variables by Student's $t$-test, and difference between proportions of binary variables by Fisher exact test, on SPSS (Chicago, IL, USA, v. 9). 
We calculated the sensitivity SN and specificity SP of an imaging appearance for a tumor type as $\mathrm{SN}=$ true-positive / (true-positive + false-negative) and SP $=$ true-negative / (true-negative + false-positive).

\section{Results}

Case series

In our combined two-site database, we had 61 children with supratentorial tumors. The children were ages 11 days to 16 years at the time of MRI, and 34/61 (55\%) were boys. Tumors included 32 gliomas (17 WHO grade 1-2 astrocytoma, 7 grade 3 astrocytoma, 6 grade 4 astrocytoma, 2 glioma not otherwise specified), 6 atypical teratoid rhabdoid tumors (ATRTs), 2 pilomyxoid astrocytoma, 8 ependymomae, 6 gangliogliomae, 3 primitive neuro-ectodermal tumors, 2 choroid plexus papillomae, and 2 meningiomae. The most common diagnosis in our series was astrocytoma.

The MRI characteristics of the supratentorial tumors in our series are summarized in Table 1. The most frequent appearance of supratentorial tumors was a solid mass (25/61, 40\%), $18(72 \%)$ of which were astrocytomas. The next most frequent appearance $(10 / 61,16 \%)$ was a cystic center associated with a mural nodule. We had 7 infiltrative tumors in our series, of which $6(86 \%)$ were astrocytomas.

Most tumors were T2-hyperintense compared to adjacent grey matter, but T2 hypointensity was seen in our choroid plexus papilloma and several high-grade tumors, including glioblastoma multiforme (2/6), PNET (1/3) and ATRT (1/6). Nearly all tumors enhanced postcontrast and had peritumoral edema. Hemorrhages were more frequent in the high-grade tumors. Restricted diffusion was seen in 20/38 (53\%) children in whom diffusion-weighted 
imaging was performed (38/61 or 62\%). Calcifications were seen on CT scan in several ependymomas, choroid plexus papillomas and astrocytomas.

We had 6 supratentorial ATRTs (10\% of our 61 patients), 3 in frontal lobes, 2 based in a lateral ventricle, and 1 in a parietal lobe. ATRTs presented in significantly younger children than astrocytomas (mean age 2.6 years vs. 9.9 years, $P<0.05$ ). ATRT size varied widely (range 16$480 \mathrm{cc}$ ). All 6 ATRTs enhanced with gadolinium, while 5/6 had central necrosis, 3/6 showed hemorrhage and 5/6 had restricted diffusion (visually and with ADC $<800 \mathrm{~mm}^{2} / \mathrm{s}$ ). Proton spectroscopy was available on 3/6 children with ATRTs. All voxels were in solid tumor and had abnormal spectra, with reduction in the $\mathrm{N}$-acetyl aspartate peak and elevated lipid and lactate peaks. None of these features distinguished ATRT from other tumor types.

A thick, wavy (irregular) heterogeneously enhancing wall encircling the lesion completely or partially was seen in 5/6 (83\%) ATRT tumors and 3/55 (5.4\%) non-ATRT tumors. For diagnosis of ATRT from this appearance, the specificity $\mathrm{SP}=0.95$, sensitivity $\mathrm{SN}=0.83$, positive predictive value $\mathrm{PPV}=0.63$ and negative predictive value $\mathrm{NPV}=0.95$, positive and negative likelihood ratios $\mathrm{LR}+=15.1, \mathrm{LR}-=0.18$. ATRT was significantly more likely to have this appearance than other tumors, $P<0.05$.

Excluding the 9/61 tumors with none of the MRI features commonly associated with high-grade diagnosis (i.e. obvious metastasis, microcystic or multicystic component, necrosis, enhancement other than thin rim, low T2 signal, hemorrhage, calcification, ill-defined border ), SN was unaffected at 0.83 and SP dropped marginally from 0.95 to 0.94 . These 9 tumors were all low-grade, but so were 19 of the 52 that showed at least one potentially high-grade feature at MRI. 
Systematic literature review

We found 17 eligible published case series (Table 2) and 14 case reports (Table 3) describing imaging appearances of pediatric supratentorial ATRT. All 17 case series included both supratentorial and intratentorial ATRTs. No study specifically examined the imaging features of supratentorial ATRTs or the difference between supratentorial and infratentorial ATRTs. The largest case series had $n=15$ supratentorial ATRT [20]. ATRTs presented mainly in younger children, though age at presentation ranged from 4 months [21] to 16 years [22]. There was data on preoperative imaging appearance of individual supratentorial ATRTs in 13/17 case series. The other four authors provided general descriptions of the tumors, as large, heterogeneous enhancing T1-hypointense tumors with cystic and hemorrhagic areas and variable T2 signal [2, 13-15].

There are only two case series that report more than 10 cases $[2,20]$. The frontal lobe is the most common location for supratentorial ATRT. Most supratentorial ATRTs have a cystic component with incidence as high as $80 \%(12 / 15)$ in the largest series [20]. They are likely to have higher attenuation on $\mathrm{CT}$ due to the high cellularity and can be associated with calcification. ATRT is an aggressive tumor, but the incidence of metastases is low at the time of imaging in most case series. However, Cheng [23] in a small series of 3 ATRTs reported 3 metastases at the time of diagnosis.

\section{Discussion}

Distribution of supratentorial tumor types in our two centres was broadly similar to other series, with ATRTs much less common than astrocytomas [24-26]. Our finding that children with ATRTs were significantly younger than those with astrocytomas confirms prior reports showing 
ATRTs generally presented in younger children $[9,27]$. The large size of supratentorial ATRTs (up to $480 \mathrm{cc}$ in our series) also confirms prior reports, in which some ATRTs occupied more than 50\% of the cerebral hemisphere [19]. Infratentorial ATRTs were smaller than supratentorial in a case series [20]. This may be due to greater supratentorial accommodation, as infratentorial tumor may quickly produce symptoms from hydrocephalus, cranial nerve or brainstem compression [28]. Supratentorial ATRT may be extension of a posterior fossa tumor that has grown upward, especially involving midbrain [29]. Most purely supratentorial ATRTs arise in cerebral hemispheres, especially the frontal lobes (Tables 2-3).

Disseminated tumors were found at diagnosis in $21-34 \%$ of patients in 3 series combining supratentorial and infratentorial ATRTs $(n=41,56$ and 52, [2, 9, 30], in just 1/15 (7\%) of patients in the largest published series of supratentorial ATRTs [20], and in 0/6 children in our data. This suggests supratentorial ATRT may have lower rate of metastasis than infratentorial ATRT. Metastases are most commonly found in leptomeninges, but the extension of supratentorial ATRTs into the skull [18-19] and extra-axial tissue [21, 31], including lung [32], have been reported, as have multifocal ATRTs [33, 34].

Most ATRTs demonstrate T1-hypointense signal and variable T2 signal, with inhomogeneous contrast enhancement, frequent necrosis and calcifications (Tables 2, 3), probably due to heterogeneous cellular populations [29]. Few supratentorial ATRTs (13-16\%) are nonenhancing $[10,20,29]$. Most authors report CT density higher than adjacent brain in the solid parts of ATRT [10, 17, 20, 23, 28, 35-37], which may correspond to the highly cellular areas seen on histology [28]. Incidence of hemorrhage in our series was 50\%, consistent with other reports (40-60\%) [17, 20, 28-29]. Cystic changes are common and are probably due to tumor necrosis. We noted from the images published in other series and in our own data that 
supratentorial ATRTs often had more prominent central cystic changes than infratentorial ATRTs, which frequently appeared primarily solid. Given the larger size of supratentorial ATRTs, this suggests the central cysts might form as the ATRTs enlarge.

Diffusion restriction was seen in 5/6 (83\%) supratentorial ATRTs in our series, consistent with other series, likely related to high nuclear-to-cytoplasmic ratios and small extracellular spaces [29, 38-39]. We had 3 ATRTs with proton MR spectroscopy available, with findings similar to other reports: elevated levels of choline and decreased $\mathrm{N}$-acetylaspartate $[29,31,40]$.

The individual findings discussed above are nonspecific and do not distinguish ATRTs from other high-grade tumors but using a combination of findings on CT and MRI can increase sensitivity and specificity. In particular, all but one (5/6) of our supratentorial ATRTs, and few other tumors, had a typical pattern of thick, wavy (irregular) heterogeneously enhancing wall surrounding a central cyst or cysts (Fig. 1), a difference that was statistically significant, specific $(\mathrm{SP}=95 \%)$ and fairly sensitive $(\mathrm{SN}=83 \%)$ for ATRT. This gave a high positive likelihood ratio $(\mathrm{LR}+=15.1)$, substantially increasing the odds of a diagnosis of ATRT if this pattern was present, and also a low negative likelihood ratio ( $L R-=0.18)$, such that the absence of this pattern substantially decreased the odds of the diagnosis being ATRT. Similarly, Arslanoglu [19] described 2 out of 3 supratentorial ATRTs having a thick, wavy (irregular) enhancing margin, and Warmuth-Metz [20] reported six ATRTs (5/6 or $83 \%$ supratentorial) having a band-like rim of strong enhancement completely surrounding a central cystic or necrotic area, and another six ATRTs (4/6 or $66 \%$ supratentorial) showed an at least partially closed band-like enhancing rim [20]. From our review of the published MR images of hemispheric supratentorial ATRTs, 8/17 case series and 4/10 case reports include cases with this appearance. Barkovich reported [41] that areas described as cysts, but having the appearance of necrosis which forms the typical irregular 
walls of the tumor are present in nearly all cases at the time of diagnosis of supratentorial ATRT. In our experience, most other supratentorial tumors are either primarily solid, have peripheral rather than a large central cysts, and/or have a smooth cyst wall. Even if the thick band of enhancement does not extend around the entire central cyst/necrosis in ATRT (Fig. 1), there is typically a complete rim of enhancement, which can be used to distinguish ATRTs from tumors with mural nodule that may not have as avidly enhancing cyst wall.

To our knowledge, this study is unique in demonstrating that the appearance of a thick, wavy (irregular) heterogeneously enhancing wall surrounding a central cystic region has high specificity for ATRTs among pediatric supratentorial brain tumors. This appearance is not pathognomonic, since some supratentorial tumors like ependymoma, PNET and choroid plexus carcinoma do infrequently present with similar appearance. However, choroid plexus carcinoma tends to have periventricular extension associated with prominent vasogenic edema [41, 42], supratentorial PNET is usually quite large at the time of presentation and sharply marginated [43] while supratentorial ependymoma occurs in older children [44, 45] and frequently calcifies $[46,47]$. These extra features also need to be considered when formulating a working differential diagnosis.

This retrospective study focusing on a rare pediatric brain tumor has limitations. Despite combining a decade of data from two tertiary pediatric centres, we still had only a small number of supratentorial ATRTs. MRI protocols varied over the decade at the two sites. We unfortunately had no diffusion-weighted imaging (DWI) in nearly half of our patients, and few children had spectroscopic data. DWI and spectroscopy are both included on current protocols at both institutions. Future studies to improve diagnostic utility of MRI for rare pediatric brain 
tumors such as ATRTs will benefit from a multicentre approach and from routine inclusion of DWI.

\section{Conclusion}

Confident diagnosis of pediatric supratentorial tumor type by MRI is rarely possible, even using diffusion-weighted imaging and proton spectroscopy. Using a combination of findings on CT and MRI can increase sensitivity and specificity. In a two-centre series of pediatric supratentorial tumors, we found an MRI appearance (thick, wavy heterogeneously enhancing wall surrounding central cyst) that strongly suggests supratentorial ATRT, especially when seen in a patient of preschool age. Although this appearance alone is not entirely diagnostic (it is also occasionally seen in supratentorial ependymoma, choroid plexus carcinoma and PNET), identifying a high probability that the tumor is an ATRT at MRI can aid in clinical decision-making. Future study could determine whether this appearance corresponds to a specific immunohistochemical or genetic profile. 


\section{References}

1. Lefkowitz IB, Rorke LB, Packer RJ (1987) Atypical teratoid tumor of infancy: definition of an entity (abstract). Ann Neurol 22:448-449

2. Rorke L B, Packer R J and Biegel J A (1996) Central nervous system atypical teratoid/rhabdoid tumors of infancy and childhood: definition of an entity. J. Neurosurg. 85, 56-65. doi: 10.3171/jns.1996.85.1.0056.

3. Beckwith JB, Palmer NF (1978) Histopathology and prognosis of Wilms tumors: results from the First National Wilms' Tumor Study. Cancer 41:1937-1948

4. Ho DM, Hsu CY, Wong TT et al (2000) Atypical teratoid/rhabdoid tumor of the central nervous system: a comparative study with primitive neuroectodermal tumor/medulloblastoma. Acta Neuropathol 99:482-488

5. Biegel JA, Zhou JY, Rorke LB et al (1999) Germ-line and acquired mutations of INI1 in atypical teratoid and rhabdoid tumors. Cancer Res 59:74-79

6. Judkins AR, Mauger J, Ht A et al (2004) Immunohistochemical analysis of hSNF5/INI1 in pediatric CNS neoplasms. Am J Surg Pathol 28:644-650

7. Samaras V, Stamatelli A, Samaras E et al (2009) Atypical teratoid/rhabdoid tumor of the central nervous system in an 18-year-old patient. Clin Neuropathol 28:1-10

8. Hashizume R, Gupta N, Berger MS et al (2010) Morphologic and molecular characterization of ATRT xenografts adapted for orthotopic therapeutic testing. Neuro Oncol 12:366-376 doi: 10.1093/neuonc/nop033.

9. von Hoff K, Hinkes B, Dannenmann-Stern E et al (2011) Frequency, risk-factors and survival of children with atypical teratoid rhabdoid tumors (AT/RT) of the CNS diagnosed 
between 1988 and 2004, and registered to the German HIT database. Pediatr Blood Cancer 57:978-985. doi: 10.1002/pbc.23236; 10.1002/pbc.23236

10. Lee IH, Yoo SY, Kim JH et al (2009) Atypical teratoid/rhabdoid tumors of the central nervous system: imaging and clinical findings in 16 children. Clin Radiol 64:256-264. doi: 10.1016/j.crad.2008.09.007

11. Morgenstern DA, Gibson S, Brown T et al (2010) Clinical and pathological features of paediatric malignant rhabdoid tumours. Pediatr Blood Cancer 54:29-34. doi: $10.1002 / \mathrm{pbc} .22231$

12. Hilden JM, Meerbaum S, Burger P et al (2004) Central nervous system atypical teratoid/rhabdoid tumor: results of therapy in children enrolled in a registry. J Clin Oncol 22:2877-2884. doi: 10.1200/JCO.2004.07.073

13. de Leon-Bojorge B, Rueda-Franco F and Anaya-Jara M (2008) Central nervous system atypical teratoid rhabdoid tumor: experience at the National Institute of Pediatrics, Mexico City. Childs Nerv Syst 24:307-312. doi: 10.1007/s00381-007-0464-9

14. Bilginer B, Bozkurt G, Akalan N et al (2006) Atypical teratoid/rhabdoid tumors. Turk Neurosurg16:77-80

15. Bambakidis NC, Robinson S, Cohen M et al (2002) Atypical teratoid/rhabdoid tumors of the central nervous system: clinical, radiographic and pathologic features. Pediatr Neurosurg $37: 64-70$

16. Fenton LZ, Foreman NK (2003) Atypical teratoid/rhabdoid tumor of the central nervous system in children: an atypical series and review. Pediatr Radiol 33:554-558. doi: $10.1007 / \mathrm{s} 00247-003-0934-5$ 
17. Parmar H, Hawkins $\mathrm{C}$, Bouffet $\mathrm{E}$ et al (2006) Imaging findings in primary intracranial atypical teratoid/rhabdoid tumors. Pediatr Radiol 36:126-132. doi: 10.1007/s00247-00500376

18. Evans A, Ganatra R, Morris SJ (2001) Imaging features of primary malignant rhabdoid tumour of the brain. Pediatr Radiol 31:631-633. doi: 10.1007/s0024710310631

19. Arslanoglu A, Aygun N, Tekhtani D et al (2004) Imaging findings of CNS atypical teratoid/rhabdoid tumors. AJNR 25:476-480

20. Warmuth-Metz M, Bison B, Dannemann-Stern E et al (2008) CT and MR imaging in atypical teratoid/rhabdoid tumors of the central nervous system. Neuroradiology 50:447-452. doi: 10.1007/s00234-008-0369-7.

21. Munoz A, Carrasco A, Munoz MJ et al (1995) Cranial rhabdoid tumor with marginal tumor cystic component and extraaxial extension. AJNR 16:1727-1728

22. Garg A, Gaikwad S, Gupta V et al (2004) Malignant rhabdoid tumour of the third ventricle. Australas Radiol 48:80-83. doi: 10.1111/j.1440-1673.2004.01251.x.

23. Cheng YC, Lirng JF, Chang FC et al (2005) Neuroradiological findings in atypical teratoid/rhabdoid tumor of the central nervous system. Acta Radiol 46:89-96

24. Mehrotra N, Shamji MF, Vassilyadi M et al (2009) Intracranial tumors in first year of life: the CHEO experience. Childs Nerv Syst 25:1563-1569. doi: 10.1007/s00381-009-0936-1.

25. Rickert CH, Paulus W (2001) Epidemiology of central nervous system tumors in childhood and adolescence based on the new WHO classification. Childs Nerv Syst 17:503-511

26. Rosemberg S, Fujiwara D (2005) Epidemiology of pediatric tumors of the nervous system according to the WHO 2000 classification: a report of 1,195 cases from a single institution. Childs Nerv Syst 21:940-944. doi: 10.1007/s00381-005-1181-x 
27. Woehrer A, Slavc I, Waldhoer T et al (2010) Incidence of atypical teratoid/rhabdoid tumors in children: a population-based study by the Austrian Brain Tumor Registry, 1996-2006. Cancer 116:5725-5732. doi: 10.1002/cncr.25540; 10.1002/cncr.25540

28. Yoon CS, Chuang S, Jay V (2000) Primary malignant rhabdoid tumor of the brain: CT and MR findings. Yonsei Med J 41:8-16

29. Meyers SP, Khademian ZP, Biegel JA et al (2006) Primary intracranial atypical teratoid/rhabdoid tumors of infancy and childhood: MRI features and patient outcomes. AJNR 27:962-971

30. Bhattacharjee M, Hicks J, Langford L et al (1997) Central nervous system atypical teratoid/rhabdoid tumors of infancy and childhood. Ultrastruct Pathol 21:369-378

31. Bing F, Nugues F, Grand S et al (2009) Primary intracranial extra-axial and supratentorial atypical rhabdoid tumor. Pediatr Neurol 41:453-456. doi: 10.1016/j.pediatrneurol.2009.07.019

32. Guler E, Varan A, Soylemezoglu F et al (2001) Extraneural metastasis in a child with atypical teratoid rhabdoid tumor of the central nervous system. J Neurooncol 54:53-56

33. Gandhi CD, Krieger MD, McComb JG (2004) Atypical teratoid/rhabdoid tumor: an unusual presentation. Neuroradiology 46:834-837. doi: 10.1007/s00234-004-1251-x

34. Fujita M, Sato M, Nakamura M et al (2005) Multicentric atypical teratoid/rhabdoid tumors occurring in the eye and fourth ventricle of an infant: case report. J Neurosurg 102 :299-302. doi: 10.3171/ped.2005.102.3.0299

35. Tez S, Koktener A, Guler G et al (2008) Atypical teratoid/rhabdoid tumors: imaging findings of two cases and review of the literature. Turk Neurosurg 18:30-34 
36. Agranovich AL, Ang LC, Griebel RW et al (1992) Malignant rhabdoid tumor of the central nervous system with subarachnoid dissemination. Surg Neurol 37:410-414

37. Hanna SL, Langston JW, Parham DM et al (1993) Primary malignant rhabdoid tumor of the brain: clinical, imaging, and pathologic findings. AJNR 14:107-115

38. Niwa T, Aida N, Tanaka M et al (2009) Diffusion-weighted imaging of an atypical teratoid/rhabdoid tumor of the cervical spine. Magn Reson Med Sci 8:135-138

39. Yuan W, Holland SK, Jones BV et al (2008) Characterization of abnormal diffusion properties of supratentorial brain tumors: a preliminary diffusion tensor imaging study. $\mathbf{J}$ Neurosurg Pediatr 1:263-269. doi: 10.3171/PED/2008/1/4/263

40. Packer RJ, Biegel JA, Blaney S et al (2002) Atypical teratoid/rhabdoid tumor of the central nervous system: report on workshop. J Pediatr Hematol Oncol 24:337-342

41. Barkovich AJ (2012) Intracranial, orbital, and neck masses of childhood. In: Pediatric neuroimaging, 5th ed. Lippincott. Williams \& Wilkins, Philadelphia, pp 708.

42. Naeini RM, Yoo JH, Hunter JV (2009) Spectrum of choroid plexus lesions in children. AJR 192:32-40. doi: 10.2214/AJR.08.1128

43. Chawla A, Emmanuel JV, Seow WT et al (2007) Paediatric PNET: pre-surgical MRI features. Clin Radiol 62:43-52. doi: 10.1016/j.crad.2006.09.008

44. Mermuys K, Jeuris W, Vanhoenacker PK et al (2005) Best cases from the AFIP: supratentorial ependymoma. Radiographics 25:486-490. doi: 10.1148/rg.252045095

45. Spoto GP, Press GA, Hesselink JR et al (1990) Intracranial ependymoma and subependymoma: MR manifestations. AJNR 11:83-91

46. Yuh EL, Barkovich AJ and Gupta N (2009) Imaging of ependymomas: MRI and CT. Childs Nerv Syst 25:1203-1213. doi: 10.1007/s00381-009-0878-7 
47. Furie DM, Provenzale JM (1995) Supratentorial ependymomas and subependymomas: CT and MR appearance. J Comput Assist Tomogr 19:518-526

48. Biswas A, Goyal S, Puri T et al (2009) Atypical teratoid rhabdoid tumor of the brain: case series and review of literature. Childs Nerv Syst 25:1495-1500. doi: 10.1007/s00381-0090903-x

49. Dang T, Vassilyadi M, Michaud J et al (2003) Atypical teratoid/rhabdoid tumors. Childs Nerv Syst 19:244-248. doi: 10.1007/s00381-003-0731-3

50. Mondal D, Jana M and Julka PK (2011) Supratentorial atypical teratoid rhabdoid tumor: An uncommon childhood tumor. J Pediatr Neurosci 6:90-91. doi: 10.4103/1817-1745.84423

51. Taneja AK, Reis F, Zanardi VA et al (2010) Meningeal presentation of an atypical teratoid/rhabdoid tumor. Neurol India 58:681-682. doi: 10.4103/0028-3886.68703

52. Donovan DJ, Smith AB and Petermann GW (2006) Atypical teratoid/rhabdoid tumor of the velum interpositum presenting as a spontaneous intraventricular hemorrhage in an infant: case report with long-term survival. Pediatr Neurosurg 42:187-192. doi: 10.1159/000091866

53. Zuccoli G, Izzi G, Bacchini E et al (1999) Central nervous system atypical teratoid/rhabdoid tumour of infancy. CT and MR findings. Clin Imaging 23:356-360

54. Howlett DC, King AP, Jarosz JM et al (1997) Imaging and pathological features of primary malignant rhabdoid tumours of the brain and spine. Neuroradiology 39:719-723

55. Honda M, Baba H, Yonekura M et al (2005) Cerebral composite atypical teratoid/rhabdoid tumor and yolk sac tumor in the frontal lobe of an infant. Case report. Neurol Med Chir (Tokyo) 45:318-321

56. Chang HK and Kim JH (2001) Classical malignant rhabdoid tumor of central nervous system in 9-year-old Korean. Yonsei Med J 42:142-146 
57. Chan KH, Mohammed Haspani MS, Tan YC et al (2011) A case report of atypical teratoid/rhabdoid tumour in a 9-year-old girl. Malays J Med Sci 18:82-86 


\section{Legend}

Fig. 1 Selected preoperative post-gadolinium T1-weighted axial MR image of 6 cases of ATRTs. a Image of an ATRT in a 2.4-year-old girl shows typical thick, wavy (irregular) heterogeneously enhancing wall completely surrounding a cystic central portion. b ATRT in a 2-year-old boy with similar findings. c An ATRT in an 8-year-old boy, with the thick lobular enhancing rim encircling most of the central cystic region. $\mathbf{d}$ An ATRT in a 0.5-year-old boy, with the thick lobular enhancing rim encircling much of the complex central cystic region, and also extending into internal septations within this. e ATRT in an 1.5-year-old girl shows thick lobular enhancing region, with minimal internal cystic change, and associated ventricular dilation. This is a smaller tumor, which likely presented early due to its location obstructing foramina of Monroe; there is little room for the central cystic change to develop. For similar reasons, posterior fossa ATRTs often appears similar to this. f An ATRT in a 1.1-year-old girl shows thick lobular enhancing rim only partially encircling the central cystic region, although there is a complete rim of thin enhancement. 
Table $1 \mathrm{MRI}$ appearance of supratentorial tumors at two pediatric tertiary centres, 2006-2011

\begin{tabular}{|c|c|c|c|c|c|c|c|}
\hline Type & $\underline{\mathbf{n}}$ & Infiltrative & Solid & & $\frac{\text { Cyst }+}{\text { nodule }}$ & Unique & $\begin{array}{l}\text { Restricted } \\
\text { diffusion }\end{array}$ \\
\hline Astrocytoma, grades 1-2 & 17 & 2 & 11 & 1 & 3 & 0 & $2 / 10$ \\
\hline Astrocytoma, grade 3 (anaplastic) & 7 & 1 & 4 & 0 & 0 & 2 & $1 / 4$ \\
\hline $\begin{array}{l}\text { Astrocytoma, grade } 4 \text { (glioblastoma } \\
\text { multiforme) }\end{array}$ & 6 & 3 & 3 & 0 & 0 & 0 & $4 / 6$ \\
\hline Glioma not otherwise specified & 2 & 0 & 1 & 0 & 0 & 1 & $1 / 2$ \\
\hline Atypical teratoid rhabdoid tumor (ATRT) & 6 & 0 & 1 & 5 & 0 & 0 & $5 / 6$ \\
\hline Pilomyxoid & 2 & 0 & 0 & c & 2 & 0 & 0 \\
\hline Primitive neuroectodermal tumor (PNET) & 3 & 0 & 2 & 1 & 0 & 0 & $2 / 2$ \\
\hline Choroid plexus papilloma & 2 & 0 & 0 & 0 & 1 & 1 & $0 / 1$ \\
\hline Ependymoma & 8 & 0 & 1 & 1 & 2 & 4 & $5 / 5$ \\
\hline Ganglioglioma & 6 & 1 & 1 & c & 2 & 2 & $0 / 2$ \\
\hline \multirow[t]{2}{*}{ Meningioma } & 2 & 0 & 1 & 0 & 0 & 1 & 0 \\
\hline & 61 & 7 & 25 & 8 & 10 & 11 & $20 / 38$ \\
\hline
\end{tabular}




\section{Table 2 Summary of published series describing imaging appearance of supratentorial ATRTs}

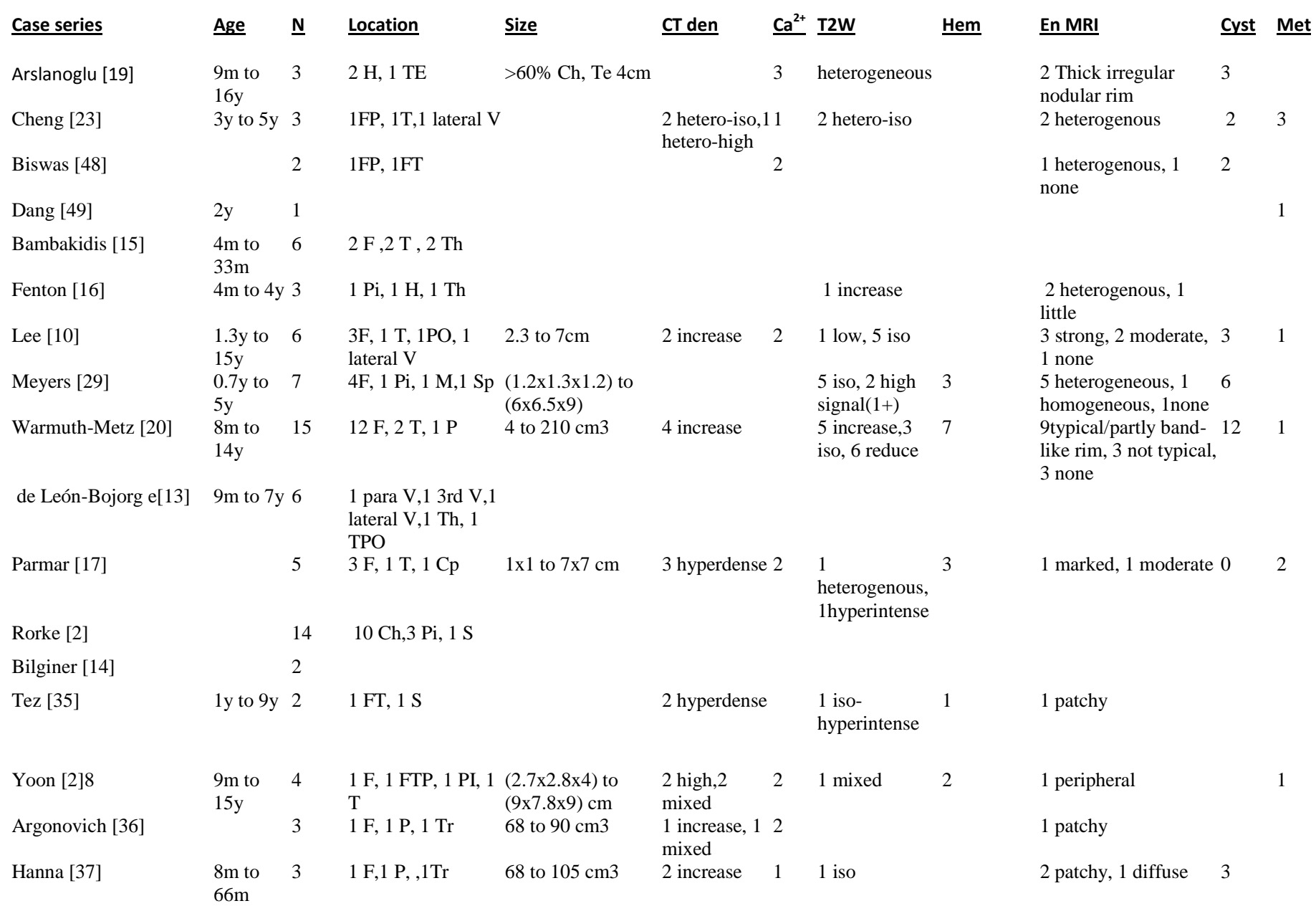

$\boldsymbol{F}$ frontal, $T$ temporal, $P$ parietal, $O$ occipital, $T h$ thalamus, $H$ hypothalamus, $V$ ventricle, $P i$ pineal, $S$ suprasellar, $\operatorname{Tr}$ trigonal, $C h$ cerebral hemispheric, $\mathrm{Sp}=$ septum pellucidum, $\mathrm{Te}=$ tectum, $\mathrm{Cp}=$ cerebral peduncle

Den density, $\mathrm{Ca}^{2+}$ calcification, Hem hemorrhage, En enhancement, Met metastasis, $m$ month, $y$ year 
Table 3 Summary of all single case studies describing the imaging appearances of supratentorial ATRTs

\begin{tabular}{|c|c|c|c|c|c|c|c|c|c|c|}
\hline Case report & Age & Location & Size & CT den & $\underline{\mathrm{Ca} 2+}$ & $\underline{\mathrm{T} W \mathrm{~W}}$ & Hem & En MRI & Cyst & Met \\
\hline Mondal [50] & $5 y$ & thalamic & & & & Heterogeneous-hyper & yes & & & no \\
\hline Taneja [51] & $11 y$ & temporal & & & & & yes & homogeneous & no & yes \\
\hline Donovan [52] & $11 w$ & velum -interpositum & & & no & & yes & & & no \\
\hline Evans [18] & $7 y$ & parieto-occipital & & increase & yes & heterogeneous & & heterogeneous & & \\
\hline Bing [31] & $1.8 \mathrm{y}$ & temporoparietal & & increase & & isodense & & heterogeneous & & no \\
\hline Ghandhi [33] & $3 m$ & chiasm and vermis & $1.6 \times 1.3 \times 1.2 \mathrm{~cm}$ & & & isodense & no & homogeneous & no & \\
\hline Zuccoli [53] & $1.7 y$ & frontoparietal & & increase & & heterogenous & & inhomogeneous & yes & no \\
\hline Howlett [54] & $5 y$ & frontoparietal & & mixed & yes & mixed & & & yes & no \\
\hline Garg [22] & $16 y$ & $3 r d$ ventricle & & increase & no & isodense & no & homogeneous & yes & no \\
\hline Honda [5]5 & $1 y$ & frontal & $8 \mathrm{~cm}$ & mixed & & & & inhomogeneous & & no \\
\hline Chang [56] & $9 y$ & temporoparietal & $9 \times 9 \times 7 \mathrm{~cm}$ & & & & & inhomogeneous & & \\
\hline Guler [32] & $6 y$ & parieto-occipital & $5 \times 5.5 \times 6.5 \mathrm{~cm}$ & & & & & & & yes \\
\hline Chan [57] & $9 y$ & temporal & & & & hypodense & & Strong enhance & yes & no \\
\hline Munoz [21] & $4 m$ & Frontal & & & yes & & & Diffuse irregular & yes & yes \\
\hline
\end{tabular}

Den density, $\mathrm{Ca}^{2+}$ calcification, Hem hemorrhage, En enhancement, Met metastasis 

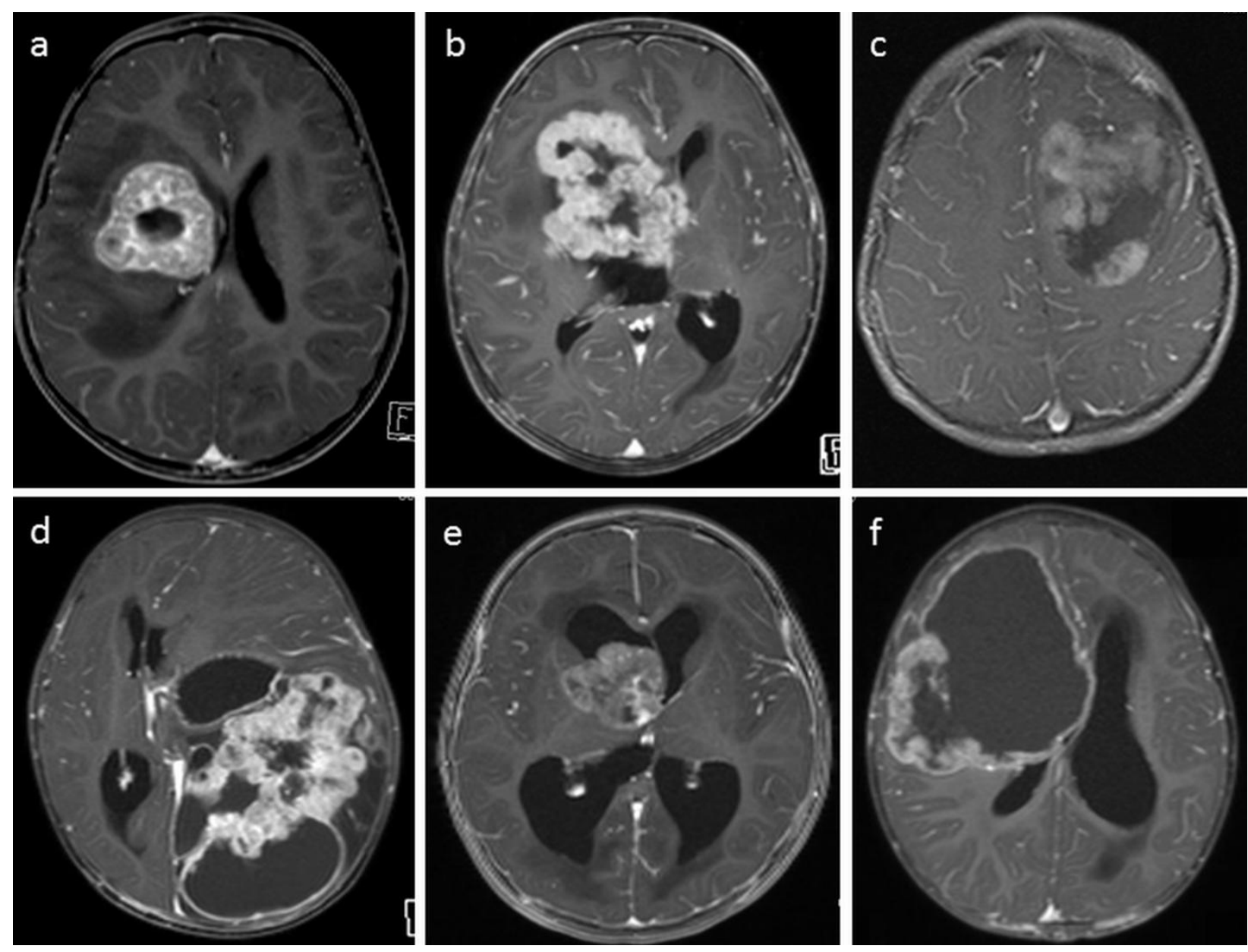